\title{
Design methodology of the primary droop voltage control for DC microgrids
}

\author{
Eduardo Prieto-Araujo, Doru Bogdan Bolboceanu, Enric Sánchez-Sánchez, Oriol Gomis-Bellmunt \\ CITCEA-UPC, ETS d'Enginyeria Industrial de Barcelona, Electrical Engineering Department \\ Universitat Politècnica de Catalunya, Barcelona, Avda. Diagonal 647, 2nd floor \\ Email: eduardo.prieto-araujo@ citcea.upc.edu
}

\begin{abstract}
In this article, a complete methodology to design the primary voltage droop control for a generic DC microgrid is proposed. First, a procedure to obtain a linear model of the complete system including the different converters inner and outer loops is detailed. Then, this linear model is analyzed using frequency domain techniques in order to ensure that the system is able to operate in a stable and secure manner. Based on the frequency analysis performed, the system droop gains are selected and tested in simulation to validate that the control design specifications are met.
\end{abstract}

Keywords-DC microgrid, droop control, voltage source converters, control design.

\section{INTRODUCTION}

The concept of microgrid is proliferating worldwide [1]. Microgrids can combine generation, storage and loads and can be controlled as an independent unit from the power system perspective [2]. These systems can typically be operated in grid-connected or in islanded mode depending on the power system state, energy market conditions and user requirements [3]. Microgrids can include a large number of components of very different nature. All these components need to be coordinated and adequately controlled [4].

Microgrids can be composed of grids of different nature, forming hybrid AC/DC systems interconnected by means of power electronics converters. Focusing on DC side of these systems, typically power electronics are in charge of maintaining the grid voltage. For this purpose, droop control is an accepted solution to regulate the DC network voltage fast, also allowing to establish a power sharing between the different converters connected to the DC grid [5]-[8]. Different implementations of the droop control [9], mainly current or power based, can be found in the literature, which can be combined to operate within the same DC grid.

This article describes a complete dynamic analysis of the DC microgrid droop control through performing a detailed study of the linearized model of the system. The selection of the droop control constants is addressed combining the obtained linear system model with advanced control design techniques to ensure that the performance specifications are met. Then, the theoretical design is validated through simulations, testing the selected droop constants under different scenarios.

\section{SYSTEM DESCRIPTION}

A generic hybrid AC/DC microgrid is presented in Fig. 1. It includes Distributed Generation (DG), an Energy Storage
System (ESS), and different loads such as an Electric Vehicle (EV) or conventional AC loads. The microgrid configuration is mainly divided in two grids of different nature, the $\mathrm{AC}$ grid side where the conventional loads are connected and the DC grid side, where the DG, EVs and ESSs are placed connected by means of DC/DC converters due to their DC inherent nature.

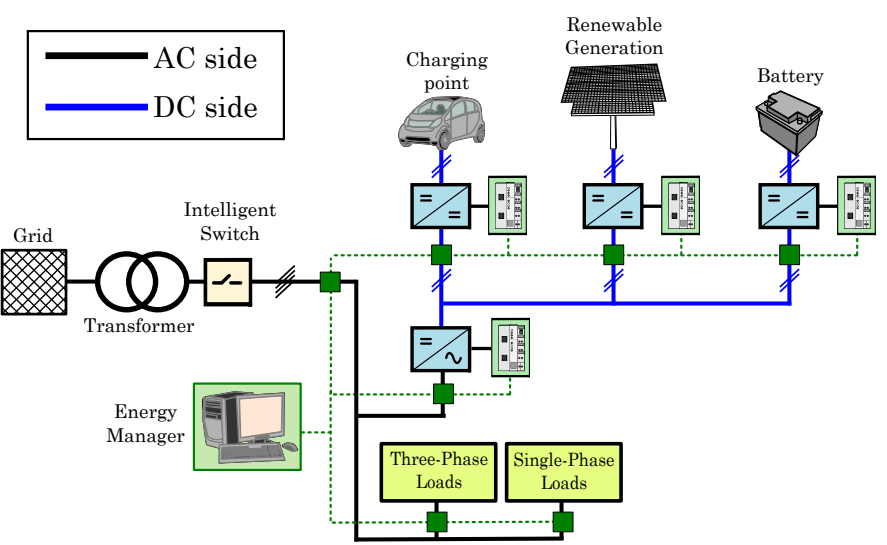

Figure 1. Hybrid AC/DC microgrid

It can be seen that different converters might be interfacing the DC microgrid side with the AC system. This fact poses a challenge on the DC voltage regulation, as it can be regulated employing different control strategies, either using a single or several converters to maintain the DC grid voltage stability. Using a single converter results in a simple control approach as the DC voltage regulator is programmed in one of the system converters and the rest can operate in power regulation mode. This strategy presents several challenges, such as the power sharing among converters, the loss of the master converter due to a temporary or a permanent outage which would cause a DC grid failure, or the loss of voltage control due to an eventual overload of the master converter.

An alternative approach is the well-known distributed droop control approach, which is able to regulate the DC grid voltage employing several converters. This controller is able to share the power between the different converters contributing to the DC voltage regulation, even without including communications, as it is implemented locally at different converters. Eventual power sharing deviations can be compensated by the upper level controllers operating in a slower time frame, which might set the voltage reference set-points. Fig. 2 shows a complete scheme of a possible control architecture following the droop control strategy. The present paper does not consider 
such an upper control layer; it is focused on the DC grid dynamic behavior. Also, it is considered that only the AC/DC converters interfacing both grids (see Fig. 1) are performing droop control to balance the DC grid voltage.

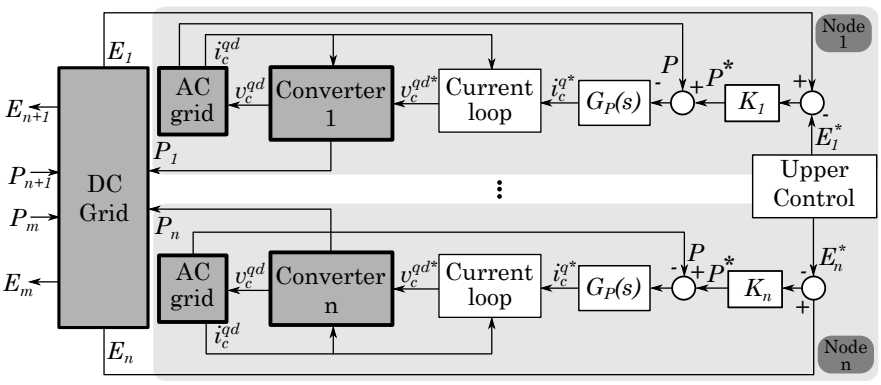

Figure 2. Complete microgrid control

As it is mentioned above, different types of droop control can be implemented. However, in this article the study is focused on the power-based droop. For the grid node $i$ the control law is expressed as:

$$
P_{i}^{*}=K_{i}\left(E_{i}-E_{i}^{*}\right)
$$

where $E_{i}$ is the measured DC voltage at the converter terminals, $E_{i}^{*}$ is the voltage reference for the droop controller (which might be different for each converter), $K_{i}$ is the droop constant and $P_{i}^{*}$ is the power reference introduced to the cascaded power loop (see Fig. 2). The converter controller includes a power loop cascaded with an inner current loop. Therefore, the power loop calculates the current set-points applied through the current controller. Regarding reactive power, different controllers can be implemented, such as a reactive power control or an $\mathrm{AC}$ voltage voltage control, depending on the system requirements.

In order to perform an adequate droop control, different dynamics must be considered such as the AC grid dynamics, the DC grid dynamics, the inherent converter dynamics and filters and the converter control dynamics. This article aims to provide a methodology able to perform a suitable design of the droop controller considering all the different dynamics that can be present in a microgrid which incorporates an internal DC grid.

\section{MICROGRID MODELING}

In this section, an equivalent model representing the dynamics of the system is detailed. Based on this model, a complete dynamic analysis including the effect of the droop control can be performed. For simplicity, the system is divided in two main parts, the AC part, including the converters and its corresponding controllers and the DC grid. The link between both parts of the model is the power flowing through the converter. It is assumed that the converters employed are conventional two-level AC/DC converters, which is a typical structure for low voltage applications.

\section{A. AC grid and converter filters}

In this section, the model of an AC/DC converter is detailed. It is considered that the grid connection is performed by an LC filter as it is shown in Fig. 3.

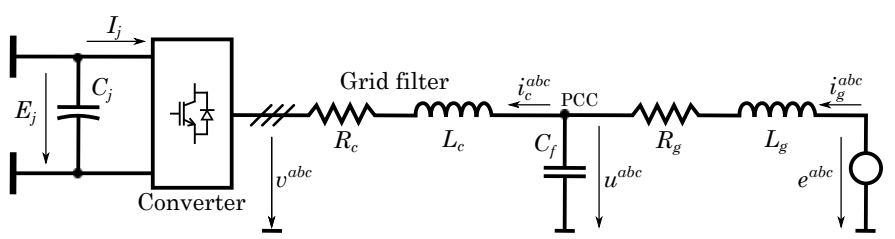

Figure 3. AC/DC converter connection to the grid

This grid connection can be represented using a statespace model in the park reference frame [10] (see Appendix), including the LC filter and the grid impedance.

$$
\frac{d x_{l c}}{d t}=\mathbf{A}_{\mathbf{l c}} x_{l c}+\mathbf{B}_{\mathbf{l c}} u_{l c}
$$

where $A_{l c}$ and $B_{l c}$ are:

$$
\begin{gathered}
\mathbf{A}_{\mathbf{l c}}=\left(\begin{array}{cccccc}
-\frac{R_{c}}{L_{c}} & -\omega & \frac{1}{L_{c}} & 0 & 0 & 0 \\
\omega & -\frac{R_{c}}{L_{c}} & 0 & \frac{1}{L_{c}} & 0 & 0 \\
-\frac{1}{C_{f}} & 0 & 0 & -\omega & \frac{1}{C_{f}} & 0 \\
0 & -\frac{1}{C_{f}} & \omega & 0 & 0 & \frac{1}{C_{f}} \\
0 & 0 & -\frac{1}{L_{g}} & 0 & -\frac{R_{g}}{L_{g}} & -\omega \\
0 & 0 & 0 & -\frac{1}{L_{g}} & \omega & -\frac{R_{g}}{L_{g}}
\end{array}\right) \\
\mathbf{B}_{\mathbf{l c}}=\left(\begin{array}{cccc}
-\frac{1}{L_{c}} & 0 & 0 & 0 \\
0 & -\frac{1}{L_{c}} & 0 & 0 \\
0 & 0 & 0 & 0 \\
0 & 0 & 0 & 0 \\
0 & 0 & \frac{1}{L_{g}} & 0 \\
0 & 0 & 0 & \frac{1}{L_{g}}
\end{array}\right)
\end{gathered}
$$

where $L_{c}$ is the filter inductance value and $R_{c}$ its parasitic resistance, $\omega$ is the frequency of the grid, $C_{f}$ is the capacitance of the capacitor filter and $L_{g}$ and $R_{g}$ are the inductance and resistance of the grid Thévenin equivalent. The state and input vectors are:

$$
\begin{aligned}
& x_{l c}=\left(i_{c}^{q}, i_{c}^{d}, u^{q}, u^{d}, i_{g}^{q}, i_{g}^{d}\right) \\
& u_{l c}=\left(v^{q}, v^{d}, e^{q}, e^{d}\right)
\end{aligned}
$$

where $i_{c}^{q d}$ are the currents flowing from the Point of Common Coupling (PCC) to the converter, $i_{g}^{q d}$ are the currents flowing to the grid, $e^{q d}$ are the grid voltages, $u^{q d}$ are the voltages at the PCC and $v^{q d}$ are the voltages applied by the converter, in the synchronous reference frame. Besides, the output vector is defined as:

$$
\Delta y_{l c}=\left(i_{c}^{q}, i_{c}^{d}, u^{q}, u^{d}, i_{g}^{d}, i_{g}^{d}, U, P_{A C}, P_{c}\right)
$$

where $U$ is the amplitude of the voltage at the PCC, $P_{A C}$ is the power flowing to the $\mathrm{AC}$ grid and $P_{c}$ is the power flowing through the converter. Note that including these magnitudes in the output vector makes the system non-linear. Then, in order to analyze the system dynamics using linear techniques, the system equations must be linearized. The linear state-space representation can be expressed as:

$$
\begin{aligned}
\frac{d \Delta x_{l c}}{d t} & =\mathbf{A}_{\mathbf{l c}} \Delta x_{l c}+\mathbf{B}_{\mathbf{l c}} \Delta u_{l c} \\
\Delta y_{l c} & =\mathbf{C}_{\mathbf{l c}} \Delta x_{l c}+\mathbf{D}_{\mathbf{l c}} \Delta u_{l c}
\end{aligned}
$$


where the matrix $\mathbf{A}_{\mathbf{l c}}$ and $\mathbf{B}_{\mathbf{l c}}$ are the matrices defined in (3) and (4). Regarding, the system output equation, matrices $\mathbf{C}_{\mathbf{l c}}$ and $\mathbf{D}_{\mathbf{l c}}$ are:

$$
\begin{gathered}
\mathbf{C}_{\mathbf{l c}}=\left(\begin{array}{cccccc}
\hline 0 & 0 & \frac{u_{0}^{q}}{U_{0}} & \frac{u_{0}^{d}}{U_{0}} & 0 & 0 \\
0 & 0 & \frac{3 i_{g 0}^{q}}{2} & \frac{3 i_{g 0}^{d}}{2} & \frac{3 u_{0}^{q}}{2} & \frac{3 u_{0}^{d}}{2} \\
\frac{3 v_{g 0}^{q}}{2} & \frac{3 v_{g 0}^{d}}{2} & 0 & 0 & 0 & 0
\end{array}\right) \\
\mathbf{D}_{\mathbf{l c}}=\left(\begin{array}{ccccc}
\frac{\mathbf{0}_{\mathbf{8 \times 4}}}{\frac{3 i_{c 0}^{q}}{2}} \frac{3 i_{c 0}^{d}}{2} & 0 & 0
\end{array}\right)
\end{gathered}
$$

where $I_{6 \times 6}$ is a $6 \times 6$ identity matrix and $\mathbf{0}_{\mathbf{8 \times 4} 4}$ is a zero matrix of 8 columns and 4 rows and $v_{0}^{q d}, u_{0}^{q d}, i_{c 0}^{q d}$ and $i_{g 0}^{q d}$ are the system variables at the linearization point.

\section{B. Phase Locked Loop (PLL)}

A Phase Locked Loop (PLL) system is required for orienting the converter controllers with the grid angle [11]. The synchronous reference frame is oriented with the d-axis voltage employing a PI regulator. The output of this controller is the estimated frequency of the grid, which is integrated to obtain the angle for the system Park transformations. The PI regulator included in the PLL is:

$$
K_{p l l}=\frac{\left(k_{p-p l l} s+k_{i-p l l}\right)}{s}
$$

where $k_{p-p l l}$ and $k_{i-p l l}$ are the proportional and integral gains of the regulator, calculated based on the amplitude of the AC voltage and the bandwidth desired for the PLL [11]. The inner dynamics of the PLL system introduce an angle deviation between the real grid angle and the estimated angle, specially during voltage transients. In order to introduce this effect into the converter linear model, the PLL tracking system can be linearized as [12], [13]:

$$
\Delta e_{\theta}=-\frac{k_{p-p l l} s+k_{i-p l l}}{s^{2}+u_{0}^{q} k_{p-p l l} s+u_{0}^{q} k_{i-p l l}} \Delta u^{d}
$$

where $e_{\theta}$ is the angle deviation between the grid angle, and the PLL estimated angle and $u_{0}^{q}$ is the voltage of the $q$ axis at the linearization point. Besides, the existing deviation between the grid real and estimated angles, must be integrated into the model variables. This effect is included as a rotation of the angle deviation between both angles. Then, two different synchronous reference frames variables are defined, the $x^{q d}\left(x^{q}\right.$ and $\left.x^{d}\right)$ variables corresponding to the synchronous reference calculated from the grid angle, and the $x^{q d c}\left(x^{q c}\right.$ and $x^{d c}$ ) variables related to the synchronous reference frame calculated from the PLL estimated angle. The transformation $\mathbf{T}_{\mathbf{c}}^{\mathbf{q d}}$ relates both references of the linear model. Its expression and its corresponding inverse are detailed in the Appendix.

\section{Current control}

The current loop is based on the conventional vector control strategy [14], based on two PI regulators $G_{C L}$ besides a decoupling loop, as it is shown in Fig. 4. The current loop model is related to $x^{q d c}$ variables, as it is affected by the potential PLL deviation. Typically, this controller is designed to track the current reference for each axis in the range of milliseconds.

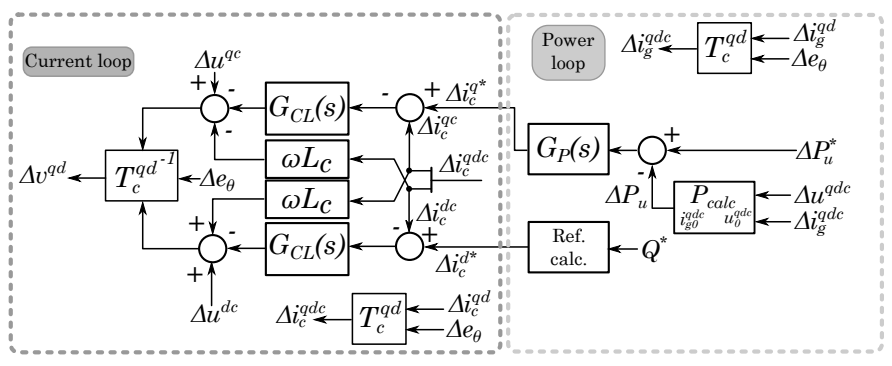

Figure 4. Current loop and power and voltage loops linearized structures

\section{Power control}

The power controller is implemented by a conventional PI regulator $\left(G_{P}\right)$, as it is shown in Fig. 4. The power feedback signal must be linear to be included into the model. Then, the linearized expression (based on the converter variables) is:

$$
\Delta P=\frac{3}{2}\left(\Delta i_{g}^{q c} u_{0}^{q}+i_{g 0}^{q} \Delta u^{q c}+\Delta i_{g}^{d c} u_{0}^{d}+i_{g 0}^{d} \Delta u^{d c}\right)
$$

The power control is typically designed to track references within the range of tens of milliseconds.

\section{E. DC grid modeling}

Regarding the DC grid model, the $\pi$-equivalent model is used to represent the system cables. The equations for a single cable are:

$$
\begin{array}{r}
\frac{d E_{i}}{d t}=\frac{1}{C_{i}}\left(I_{i}-I_{i j}\right)=\frac{1}{C_{i}}\left(\frac{P_{i}}{E_{i}}-I_{i j}\right) \\
\frac{d E_{j}}{d t}=\frac{1}{C_{j}}\left(I_{i j}-I_{j}\right)=\frac{1}{C_{j}}\left(I_{i j}-\frac{P_{j}}{E_{j}}\right) \\
E_{i}-E_{j}=R_{i j} I_{L i j}+L_{i j} \frac{d I_{i j}}{d t}
\end{array}
$$

where $E_{i}$ and $E_{j}$ are the voltages at the equivalent capacitors, $I_{i}$ and $I_{j}$ are the currents flowing through the converter, $P_{i}$ and $P_{j}$ are the input/output power flowing through the converters, $I_{L i j}$ is the current flowing through the line, $C_{i}$ and $C_{j}$ are the equivalent capacitances at both sides of the DC link and $R_{i j}$ and $L_{i j}$ are the equivalent cable resistance and inductance of the $\pi$-equivalent model. Note that the current input variables are obtained based on the division of power and voltage, thus it must be linearized as:

$$
I_{i}=\frac{P_{i}}{E_{i}} \approx \frac{\Delta P_{i}}{E_{i 0}}-\frac{P_{i 0}}{E_{i 0}^{2}} \Delta E_{i}
$$

where $\Delta E_{i}$ and $\Delta P_{i}$ are linearized variables representing the voltage at the node $i$ and the power flowing through the converter $i$, respectively. Also, $E_{i 0}$ and $P_{i 0}$ are the power and the voltage at the linearization point. Finally, combining the previous expressions, the DC link state-space representation can be obtained. Further, combining different DC grid cable models with the DC-link capacitors of the converters, an equivalent linear state-space model of the grid can be obtained. 


\section{CASE STUDY}

The droop control design methodology is explained using the case study microgrid shown in Fig. 5. It consists of a Photovoltaic (PV) generation system (PV module and DC/DC converter) which is connected to two different AC/DC converters, which are interfacing the AC grid at two different points of connection. The parameters of the system are shown in Tables I and II.

Table I. PARAMETERS OF THE DC GRID

\begin{tabular}{ccc}
\hline DC grid parameters & Value & Units \\
\hline Cable 1 resistance $R$ & 1 & $\Omega$ \\
Cable 1 inductance $L$ & 0.2 & $\mathrm{mH}$ \\
Cable 2 resistance $R$ & 1 & $\Omega$ \\
Cable 2 inductance $L$ & 0.2 & $\mathrm{mH}$ \\
Converter 1 power $S_{1}$ & 10 & $\mathrm{kVA}$ \\
Converter 2 power $S_{2}$ & 10 & $\mathrm{kVA}$ \\
Converter 3 power $P_{3}$ & 10 & $\mathrm{~kW}$ \\
Reference voltage $E^{*}$ & 800 & $\mathrm{~V}$ \\
\hline
\end{tabular}

Table II. PARAMETER OF THE AC/DC CONVERTERS

\begin{tabular}{ccc}
\hline AC side parameters & Value & Units \\
\hline Nominal Voltage $V_{a c}$ & 400 & $\mathrm{~V}$ \\
Grid Thvenin Rn/Xn ratio & 0.9 & - \\
Coupling inductance $L_{c}$ & 5.4 & $\mathrm{mH}$ \\
Coupling resistance $R_{c}$ & 0.5 & $\Omega$ \\
Capacitor filter impedance $X_{f}$ & 4.7 & $\mathrm{uF}$ \\
DC bus capacitor $C_{d c}$ & 1 & $\mathrm{mF}$ \\
\hline
\end{tabular}

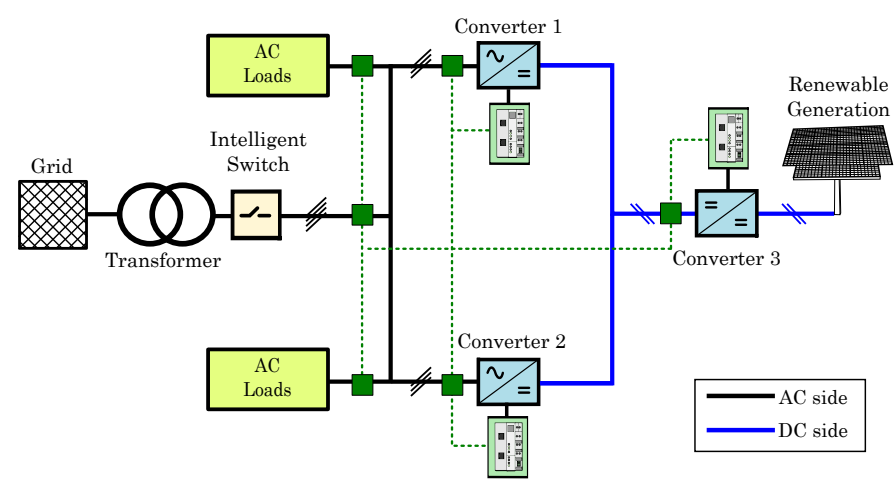

Figure 5. Case study microgrid

Based on the different parts of the model that have been presented, a methodology to perform a sequential design for the different controllers involved in the DC grid is applied. As the droop control behavior not only depends on the DC or AC grid dynamics, but also on the inner control loops of the converter (see Fig. 4), a specific design for the current and power loop is also presented, and both dynamics are further considered during the droop tuning process.

\section{A. Current control loop}

The inner control current is based on vector control in the synchronous reference frame, tuned by IMC control technique [14]. Therefore, two PI regulators $\left(G_{C L}\right)$ plus a decoupling loop are employed to track the $x^{q d}$ current references in a defined time $\tau$, following a first order system response:

$$
G_{C L}(s)=\frac{k_{p} s+k_{i}}{s}, \quad k_{p}=\frac{L_{c}}{\tau}, \quad k_{i}=\frac{R_{c}}{\tau}
$$

The control time constant $\tau$ is set to $1 \mathrm{~ms}$. Fig. 6 shows a response comparison of the complete simulation model versus the linear model derived based on the system equations, showing an adequate behavior.
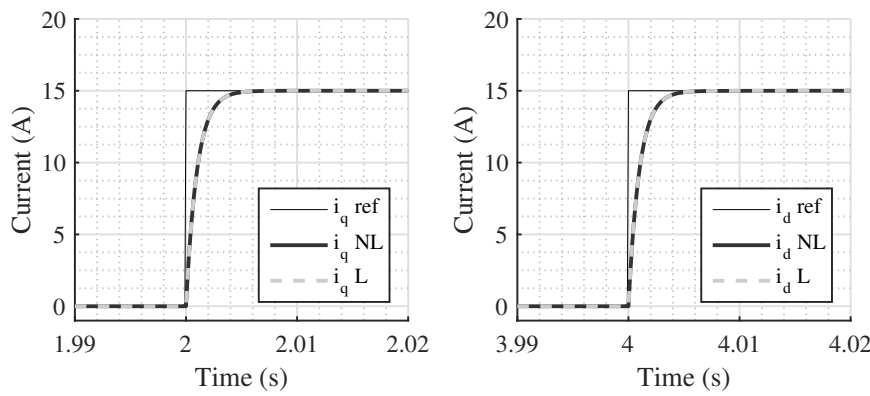

Figure 6. Current loop response (active and reactive current). Comparison between complete and linear model.

\section{B. Power loop}

A conventional PI controller $G_{P}$ is used to track power references. As the power loop is receiving references from the droop voltage loop (see Fig. 2), it needs a relatively fast response to avoid large deviations of the DC voltage. The parameters of the controller are obtained using optimization robust control techniques [15], [16]. The inputs for the optimization are basically the desired frequency response for the controller expressed as an objective transfer function. Then, the optimization algorithm is executed to select the PI parameters based on the frequency domain requirements.

The power controller settling time is set to $100 \mathrm{~ms}$. Fig. 7 shows a response comparison of the complete simulation model versus the linear model derived based on the system equations, showing that both models match properly.

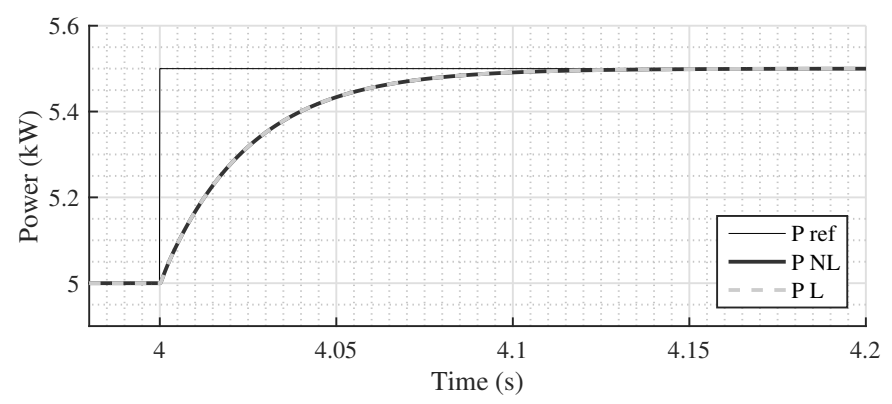

Figure 7. Power loop response. Comparison between complete and linear model.

\section{Droop control}

The droop control is designed considering the previously described dynamics together with the DC network dynamics. A complete linear model is derived combining the different subsystems described as it is shown in Fig. 8.

Once the linear model has been implemented, the requirements for the droop control must be defined. For the case study, the control specifications are:

- Equal power sharing between both converters. 


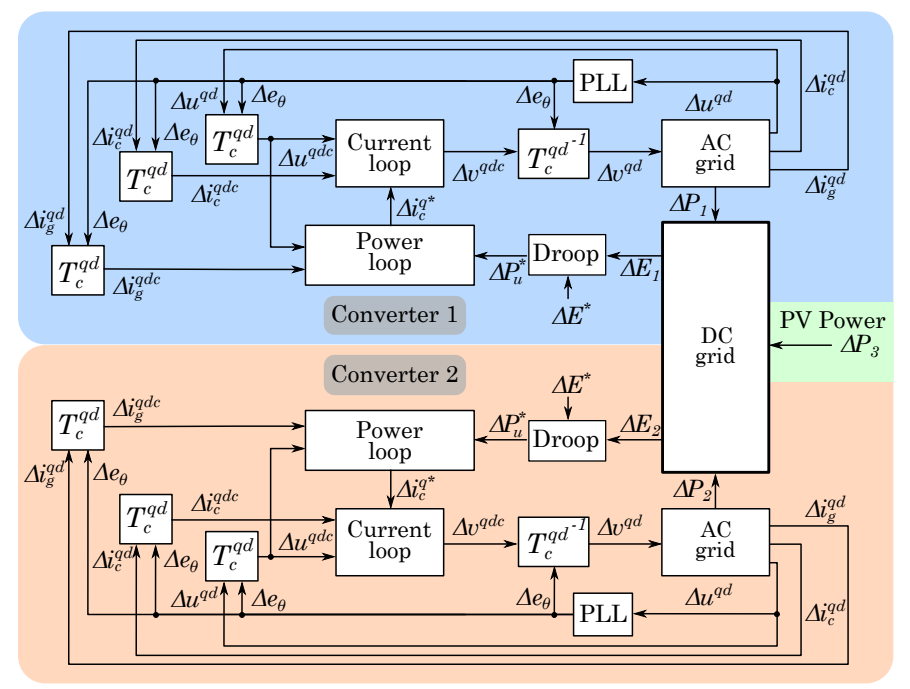

Figure 8. Complete linear model scheme of the case study

- Maximum DC voltage deviation: $10 \%$ at the different nodes.

- Avoid over-currents in any of the converters. A maximum transient deviation of $10 \%$ is allowed above the converters' nominal power value.

Based on these specifications, a frequency response analysis of the complete system is carried out to find the suitable droop constants that fulfill the required specifications. The control design procedure consists on imposing gain limitations on the frequency response of selected transfer functions of the system.

In this case, as the system is a Multiple Input Multiple Output (MIMO) system, the Singular Value Decomposition (SVD) technique can be used to obtain the maximum gains over frequency. Two main transfer functions are analyzed during the design procedure:

- TF1. Transfer function matrix relating the voltage errors at the droop controlled nodes $\left(e_{1}\right.$ and $\left.e_{2}\right)$ with the power injected by the PV $\left(P_{3}\right)$.

- TF2. Transfer function matrix relating the control action at both converters $\left(P_{r e f 1}\right.$ and $P_{r e f 2}$ with the power injected by the $\mathrm{PV}\left(P_{3}\right)$.

Then, knowing in advance the maximum allowed voltage deviation $e_{\max 1}$ and $e_{\max 2}$, and the maximum PV power input $P_{\max 3}$ a gain constraint can be imposed to the frequency response of TF1, calculated as

$$
\begin{gathered}
\bar{\sigma}(\mathrm{TF} 1(0)) \leq \frac{\|e(0)\|_{2}}{\|w(0)\|_{2}}=20 \log _{10}\left(\frac{\sqrt{\left(e_{\max 1}^{2}+e_{\max 2}^{2}\right)}}{P_{\max 3}}\right)= \\
=20 \log _{10}\left(\frac{\sqrt{(800 \cdot 0.1)^{2} \cdot 2}}{10 \cdot 10^{3}}\right)=-38.92 \mathrm{~dB}
\end{gathered}
$$

Analogously, a maximum gain deviation can be imposed to $\mathrm{TF} 2$, calculated as

$$
\begin{gathered}
\bar{\sigma}(\mathrm{TF} 2(0)) \leq \frac{\|u(0)\|_{2}}{\|w(0)\|_{2}}=20 \log _{10}\left(\frac{\sqrt{\left(P_{r e f 1}^{2}+P_{r e f 2}^{2}\right)}}{P_{\max 3}}\right)= \\
=20 \log _{10}\left(\frac{\sqrt{\left(10 \cdot 10^{3} \cdot 1.1\right)^{2} \cdot 2}}{10 \cdot 10^{3}}\right)=3.83 \mathrm{~dB}
\end{gathered}
$$

Both constraints can be relaxed or stressed with frequency due to the limitations of the power loop controller. It is assumed that it cannot act over $40 \mathrm{rad} / \mathrm{s}$, based on its previous design. Thus, on the one hand TF1 constraint is relaxed to allow error deviations at frequencies further than $40 \mathrm{rad} / \mathrm{s}$. On the other hand, TF2 constraint is stressed to avoid acting with the power loop above $40 \mathrm{rad} / \mathrm{s}$. Both constraints can be seen in Fig. 9.
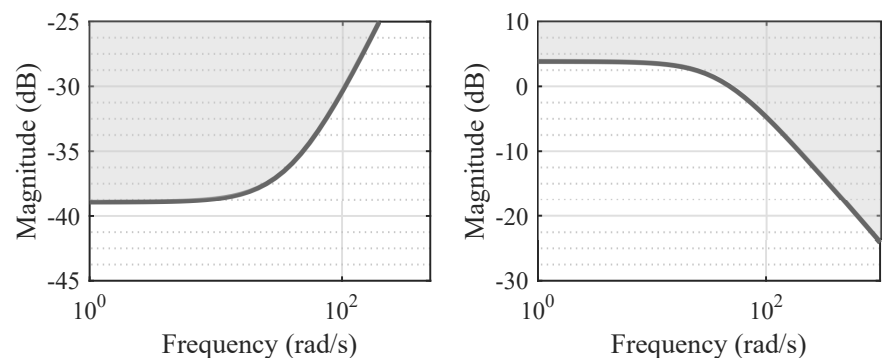

Figure 9. Design frequency constraints for TF1 and TF2.

Once the control requirements have been established, the control tuning can performed. The droop controller can be expressed, in its multi-variable form, as:

$$
K=\left(\begin{array}{cc}
K_{1} & 0 \\
0 & K_{2}
\end{array}\right)
$$

In principle, the power must be shared equally by both converters, which might be imposed as a restriction to the system design.

Then, based on the suitable areas defined, a control optimization routine is executed to find the most suitable droop controller that would fulfill the required criteria (see Fig. 9). The maximum power limitation is set as a hard goal in order to avoid exceeding the current limitations of the converter and the error limitation is maintained as a soft goal, for the optimization routine. The control optimization is based on the theory developed in [16] which is able to select the most adequate $K_{1}$ and $K_{2}$ to match the specifications. The optimization extracts a value of $85 \mathrm{~W} / \mathrm{V}$ for both droop controllers, ensuring that the system meets the defined criteria as it is shown in Fig. 10.

In order to validate the design, a first simulation model showing the system response to an increment of the PV injected power of a $+10 \%$ respect to the nominal value (linearization point) is carried out. Fig. 11 shows an adequate transient behavior of the DC network voltages, converters' power and AC currents in qd frame, after the power step. It can also be seen that both the linear and the complete model present the same dynamics, confirming that the linear model is suitable for designing the droop control. 

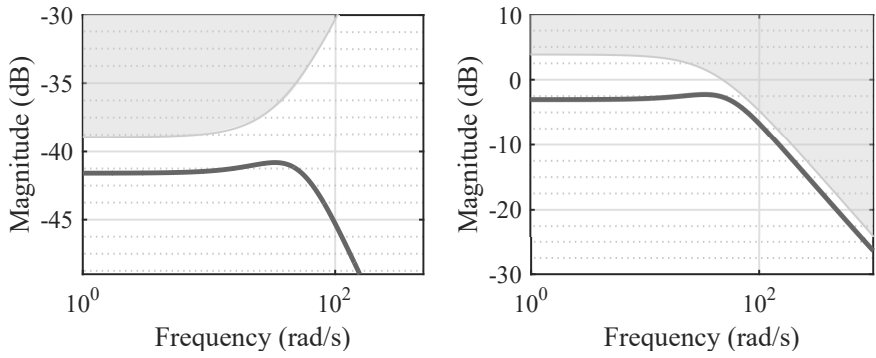

Figure 10. Final design output. Frequency response of TF1 and TF2.
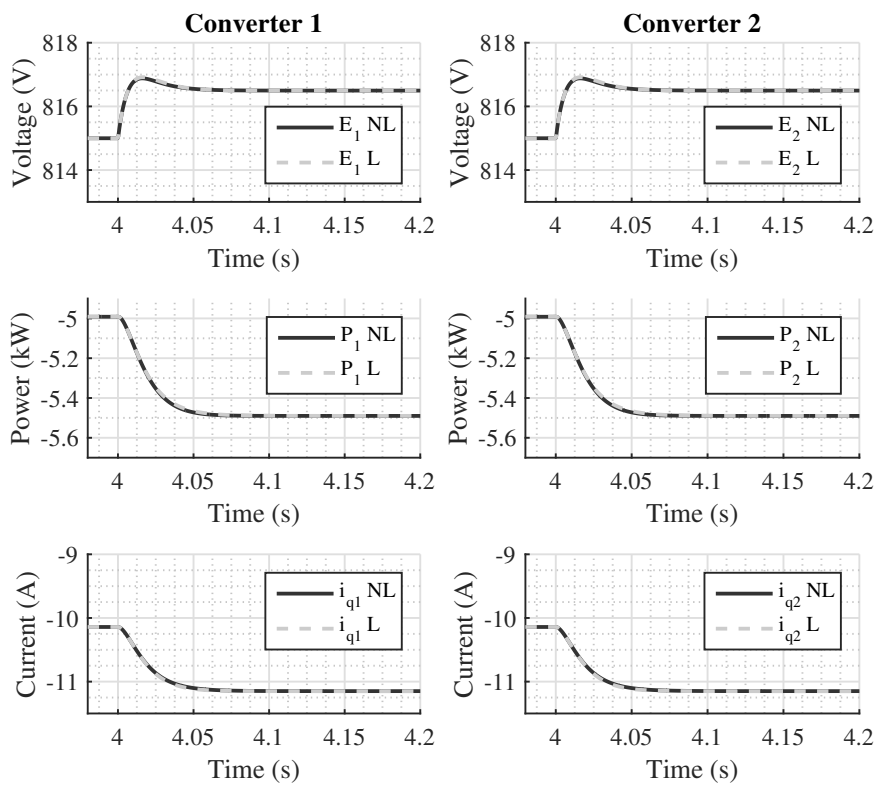

Figure 11. Power step change response. Complete (NL) and Linear (L) model comparison.

Apart from the model verification, a second simulation is carried out to show the network response to a PV power step change evolution from 0 to the nominal power value $10 \mathrm{~kW}$. Figs. 12 and 13 show the corresponding results, confirming the adequate response of the droop controller design, avoiding large over-voltages and over-currents, even considering a fast step power income.

\section{CONClusions}

A DC voltage droop design methodology considering the different dynamics present in a DC microgrid is presented. The methodology includes a procedure for obtaining a linearized model of the complete system. Also, a design example for the current loop and the power loop is provided. The methodology is applied to a DC microgrid example. First, the inner dynamics of the converter are established. Then, using advanced control techniques applied to the obtained linear model, the design of the DC droop voltage control is carried out. The results of the design procedure are validated through simulations showing an adequate system response.
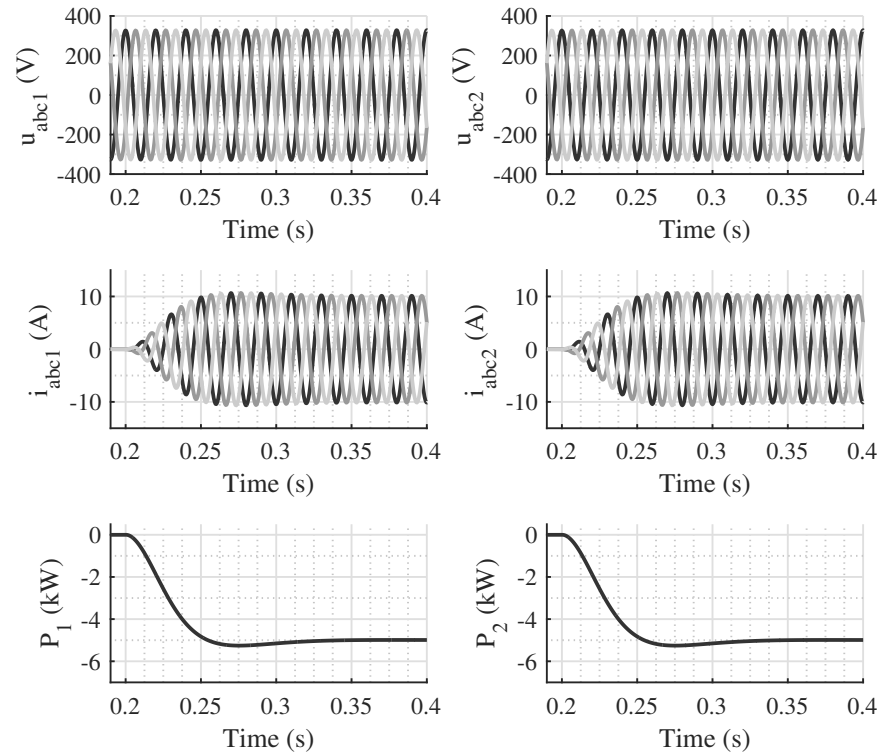

Figure 12. System response to a nominal power step change. AC system variables.
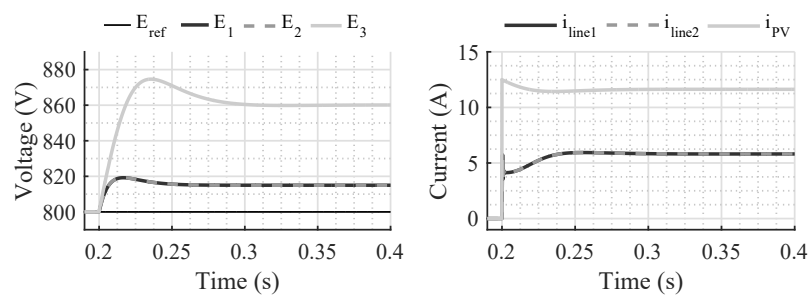

Figure 13. System response to a nominal power step change. DC system variables.

\section{APPENDIX}

Park transformation matrix:

$$
\mathbf{T}(\theta)=\frac{3}{2}\left(\begin{array}{ccc}
\cos (\theta) & \cos \left(\theta-\frac{2 \pi}{3}\right) & \cos \left(\theta+\frac{2 \pi}{3}\right) \\
\sin (\theta) & \sin \left(\theta-\frac{2 \pi}{3}\right) & \sin \left(\theta+\frac{2 \pi}{3}\right) \\
\frac{1}{2} & \frac{1}{2} & \frac{1}{2}
\end{array}\right)
$$

The transformation $\mathbf{T}_{\mathbf{c}}^{\mathbf{q d}}$ relates both references of the linear model is:

$$
\Delta x^{q d c}=\mathbf{T}_{\mathbf{c}}^{\mathbf{q d}}\left(\Delta x^{q}, \Delta x^{d}, \Delta e_{\theta}\right)^{T}
$$

where $\mathbf{T}_{\mathbf{c}}^{\mathbf{q d}}$ is:

$\mathbf{T}_{\mathbf{c}}^{\mathbf{q d}}=\left(\begin{array}{ccc}\cos \left(e_{\theta_{0}}\right) & -\sin \left(e_{\theta_{0}}\right) & -\sin \left(e_{\theta_{0}}\right) x_{0}^{q}-\cos \left(e_{\theta_{0}}\right) x_{0}^{d} \\ \sin \left(e_{\theta_{0}}\right) & \cos \left(e_{\theta_{0}}\right) & \cos \left(e_{\theta_{0}}\right) x_{0}^{q}-\sin \left(e_{\theta_{0}}\right) x_{0}^{d}\end{array}\right)$

where $e_{\theta_{0}}$ is the angle error deviation value at the linearization point and $x_{0}^{d}$ and $x_{0}^{q}$ are the $x^{q d}$ components magnitudes around at the linearization point. Also, the inverse transformation can be defined as:

$$
\Delta x^{q d}=\mathbf{T}_{\mathbf{c}}^{\mathbf{q d}^{-1}}\left(\Delta x^{q c}, \Delta x^{d c}, \Delta e_{\theta}\right)^{T}
$$

where $\mathbf{T}_{\mathbf{c}}^{\mathbf{q d}^{-1}}$ is:

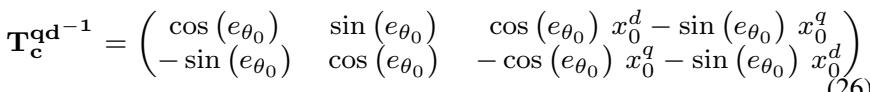




\section{ACKNOWLEDGMENTS}

This work was supported in part by the Spanish Ministry of Economy and Competitiveness under Project and ENE201567048-C4-1-R in part by the European Regional Development Fund.

\section{REFERENCES}

[1] R. Lasseter, "Microgrids," in IEEE Power Engineering Society Winter Meeting, 2002., vol. 1, 2002, pp. 305-308.

[2] N. Hatziargyriou, H. Asano, R. Iravani, and C. Marnay, "Microgrids," IEEE power and energy magazine, vol. 5, no. 4, pp. 78-94, 2007.

[3] D. E. Olivares, A. Mehrizi-Sani, A. H. Etemadi, C. A. Canizares, R. Iravani, M. Kazerani, A. H. Hajimiragha, O. Gomis-Bellmunt, M. Saeedifard, R. Palma-Behnke et al., "Trends in microgrid control," IEEE Trans. on smart grid, vol. 5, no. 4, pp. 1905-1919, 2014.

[4] E. Planas, A. G. de Muro, J. Andreu, I. Kortabarria, and I. M. de Alegra, "General aspects, hierarchical controls and droop methods in microgrids: A review," Renewable and Sustainable Energy Rev., vol. 17, no. 0, pp. 147-159, 2013.

[5] J. M. Guerrero, J. C. Vasquez, J. Matas, L. G. de Vicuna, and M. Castilla, "Hierarchical control of droop-controlled ac and dc microgrids. a general approach toward standardization," IEEE Trans. on Ind. Electron., vol. 58, no. 1, pp. 158-172, Jan 2011.

[6] S. Augustine, M. K. Mishra, and N. Lakshminarasamma, "Adaptive droop control strategy for load sharing and circulating current minimization in low-voltage standalone de microgrid," IEEE Trans. on Sustain. Energy, vol. 6, no. 1, pp. 132-141, 2015.

[7] S. Augustine, N. Lakshminarasamma, and M. K. Mishra, "Control of photovoltaic-based low-voltage dc microgrid system for power sharing with modified droop algorithm," IET Power Electronics, vol. 9, no. 6, pp. 1132-1143, 2016.

[8] X. Li, L. Guo, S. Zhang, C. Wang, Y. W. Li, A. Chen, and Y. Feng, "Observer-based dc voltage droop and current feed-forward control of a de microgrid," IEEE Trans. on Smart Grid, 2017.

[9] F. Thams, R. Eriksson, and M. Molinas, "Interaction of droop control structures and its inherent effect on the power transfer limits in multiterminal vsc-hvdc," IEEE Trans. on Power Del., vol. 32, no. 1, pp. 182-192, Feb 2017.

[10] C. Ong, Dynamic Simulation of Electric Machinery: Using MATLAB/SIMULINK. Prentice Hall PTR, 1998.

[11] S.-K. Chung, "A phase tracking system for three phase utility interface inverters," IEEE Trans. Power Electron., vol. 15, no. 3, pp. 431-438, 2000.

[12] L. Zhang, "Modeling and control of VSC-HVDC links connected to weak ac systems," Ph.D. dissertation, KTH, Electrical Machines and Power Electronics, 2010.

[13] L. Harnefors, M. Bongiorno, and S. Lundberg, "Input-admittance calculation and shaping for controlled voltage-source converters," IEEE Trans. Ind. Electron., vol. 54, no. 6, pp. 3323-3334, Dec. 2007.

[14] L. Harnefors and H.-P. Nee, "Model-based current control of AC machines using the internal model control method," IEEE Trans. Ind. Appl., vol. 34, no. 1, pp. 133-141, Jan. 1998.

[15] A. Egea-Alvarez, S. Fekriasl, F. Hassan, and O. Gomis-Bellmunt, "Advanced vector control for voltage source converters connected to weak grids," IEEE Trans. Power Syst., vol. 30, no. 6, pp. 3072-3081, Nov. 2015.

[16] P. Apkarian and D. Noll, "Nonsmooth H-infinity synthesis," IEEE Trans. Autom. Control, vol. 51, no. 1, pp. 71-86, Jan. 2006. 